\title{
The Research of Wind Turbine Airfoils Aerodynamic Characteristics Effects Based on Numerical Simulation
}

\author{
Chong Meng ${ }^{1, a}$
}

\author{
${ }^{1}$ North China Electric Power University . Huadian Road No. 689. Baoding City in Hebei ,China \\ awamengchong@163.com
}

Keywords: geometric parameter, aerodynamic characteristics, numerical simulation, mechanism analysis.

\begin{abstract}
With the development of large-scale wind turbine, the limitation of the classical aerodynamic characteristics of wind turbine research methods has become more sophisticated, and the improvement of CFD numerical simulation technology has provided a new research method for wind turbine aerodynamic characteristics. By mean of FLUENT software, we did a quantitative investigation on the influence of the same airfoil at different angles of attack to the aerodynamic characteristics and compared it with the results of the classical methods. It confirmed the accuracy and reliability of CFD numerical simulation method which could be widely used as an aerodynamic research related aid.
\end{abstract}

\section{Introduction}

Currently, as the large-scale wind turbine develops, the study of aerodynamic performance and structural properties of the whole airfoil has become more important. In the 1880s, Richard Froude proposed blade element theory, and later momentum-blade element theory was developed on its foundation which was widely used in small wind turbines[1]. With the increase in unit capacity of wind turbine, the accuracy decreases. In recent years, with the development of computational fluid dynamics and computer hardware, numerical simulation has become an important research method of knowing the flow law which is simple, controllable and accurate[2]. It also has provided a way for understanding the wind turbine airfoil aerodynamic performance.

Therefore, in this article, CFD numerical simulation will be used to explore the effects of different angles of attack to the wind turbine airfoils aerodynamic characteristics, which will be compared with the classical results to prove the accuracy and reliability of CFD numerical stimulation.

\section{Aerodynamic performance parameters of wind turbine airfoils}

\subsection{Lift coefficient and Drag coefficient}

Lift coefficient is the ratio of the object by the lift and the product of gas flow pressure and the reference area, and drag coefficient refers to the ratio of the object by the resistance and the product of gas flow pressure and the reference area, both of which are dimensionless quantity[3]. Their expressions are as follows:

$$
\begin{aligned}
& C_{L}=\frac{L}{\frac{1}{2} \rho v^{2} S} \\
& C_{D}=\frac{D}{\frac{1}{2} \rho v^{2} S}
\end{aligned}
$$

$\mathbf{L}$ is the lift of the entire blade suffered. $\mathbf{D}$ is the resistance of the entire blade. $\mathbf{S}$ refers to the leaf area. $\mathbf{P}$ is the airflow density. $\mathbf{V}$ is the airflow velocity. 


\subsection{Torque coefficient}

Torque is generated by the gas flowing to the blade, which is also the main coefficient reflecting the wind turbine performance. In the design of wind turbine, to get large output power, large torque should be obtained as much as possible.

$$
C_{m}=\frac{M}{\frac{1}{2} \rho v^{2} t S}
$$

M: torque the whole blade suffered, S: leaf area

P: airflow density, V: airflow velocity

\section{Numerical simulation based on FLUNET}

\section{1 model preprocessing}

We did research on airfoil NACA83024, and from right to left set the airflow area at 5 times the chord length. Airflow velocity was $0.8 \mathrm{Mach}$, and angle of attack was $14^{\circ}$. Because of its simple graphics, we used Gambit quadrilateral mesh topology. The results are as follows:

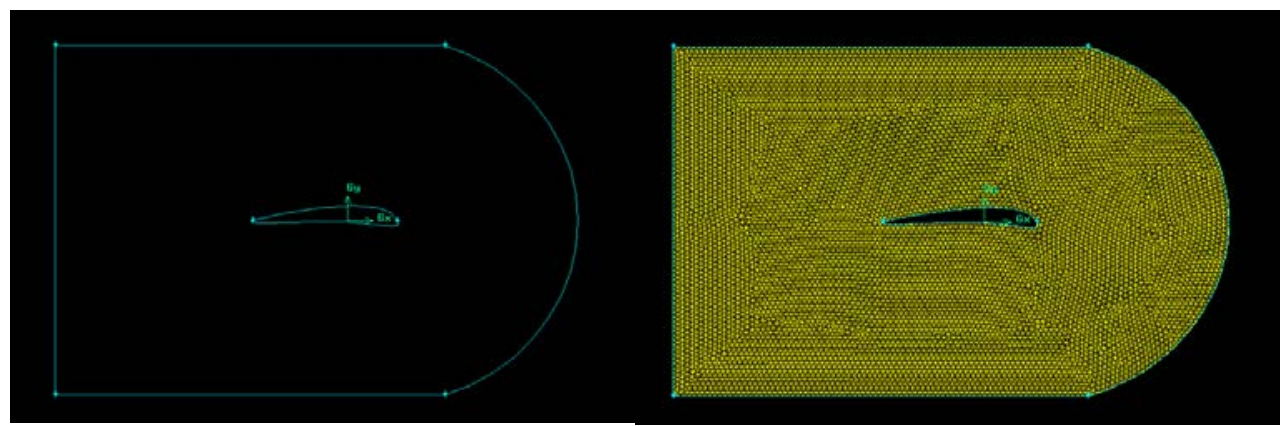

Fig.2 Computational domain selection

Fig.3 mesh generation

\subsection{Model assumption}

(1) Research object has infinite wingspan and chord and airfoil do not change. Each section of fluid flow around airfoil is the same, which is a tow-dimensional motion.

(2) Ignoring the impact of any solid wall beyond airfoil and only thinking about airfoil's movement in the still fluid, we can draw a conclusion that uniform flow flows around airfoil.

(3) The flow gas is an ideal gas which meets the equation of ideal gas state.

\subsection{Model calculation}

(1) Solver selection: as the research object is a high-speed aerodynamic problem with shock wave, so we use pressure roadbed solver and Colin gaussian function based on node to get more precise gradient, so that we can calculate the resistance more precisely.

(2) The selection of turbulence model: setting the S-A model as an output method based on stress/vortex. S-A model is a relatively simple equation model which is to solve a transport equation related to eddy viscosity, more suitable for the flow problem with wall limit. Good calculated results can be given when it works in solving the boundary layer problem of adverse pressure gradient. Therefore, it is often used in aerodynamic problems.

(3)Boundary condition: Setting the flow field boundary is far pressure sideline to define a compressible free flow in the distance.

\subsection{The simulation results and analysis}

We used the second order upwind equation to calculate and properly control relaxation factor, iterating 200 times, then obtained the residual iteration curve and aerodynamic characteristics curve. 

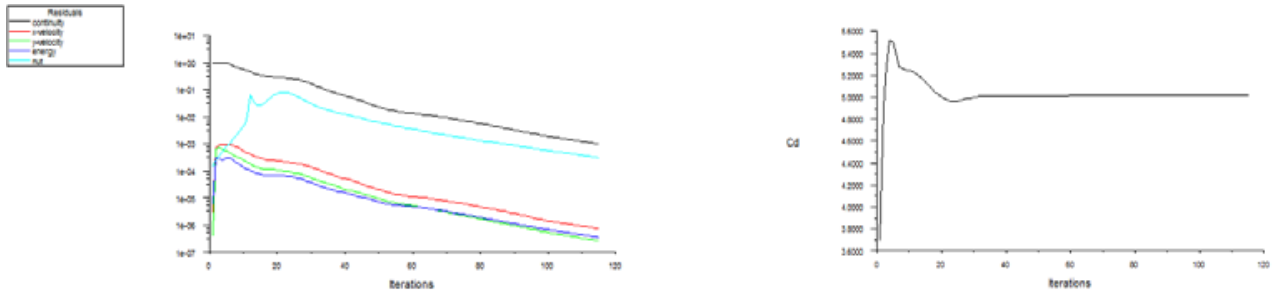

Fig.4 Iterative error curve

Fig.5 Drag coefficient curve over the iterative process
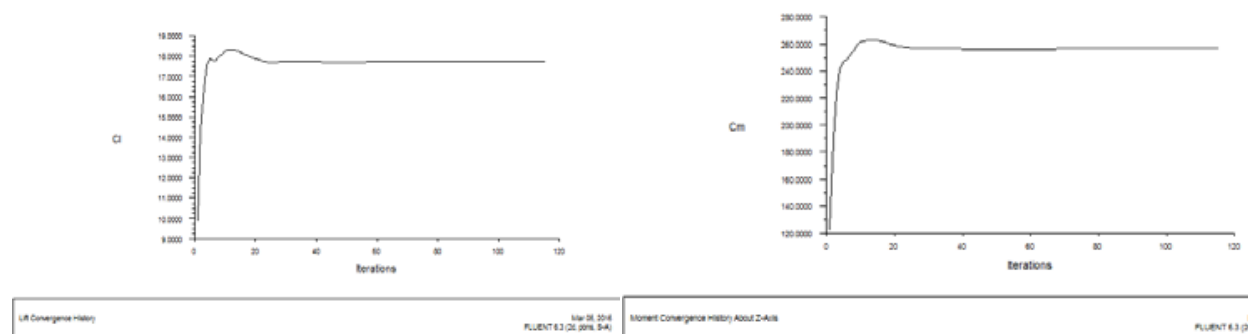

Fig.6 Lift coefficient curve over the iterative process

Fig.7 torque coefficient curve over the iterative process

Regarding the parameters of flow process of gas as reference, we can obtain the airfoil drag coefficient $C_{D}=5$, Lift coefficient $C_{L}=17.8$, Torque coefficient $C_{m}=257$

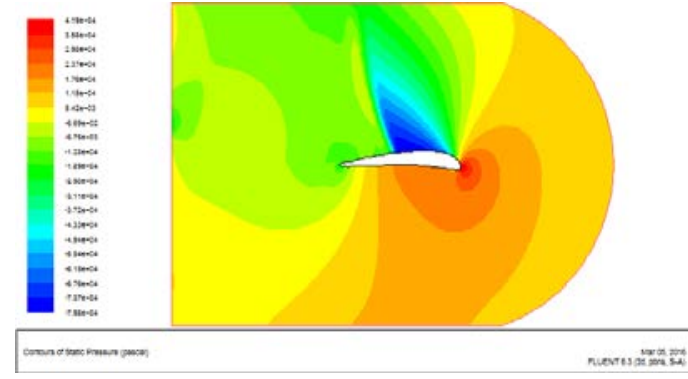

Fig.8 pressure nephogram

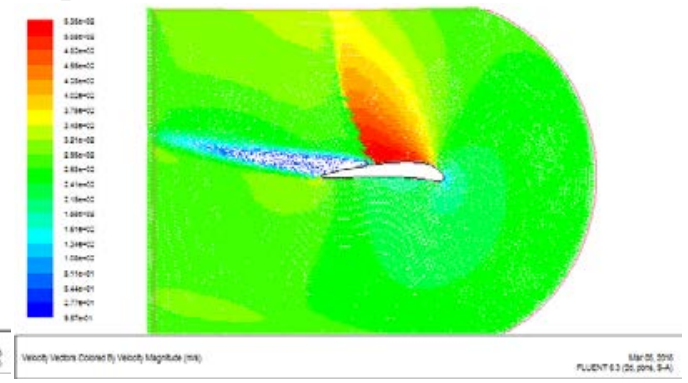

Fig.9 velocity vector

In the pressure nephogram, we can clearly see the low pressure area formed above the airfoil. And the lift is generated under the joint action of high pressure beneath the airfoil. While in the velocity vector, it shows there are speed and low-speed area around the airfoil. So we amplify these zones.

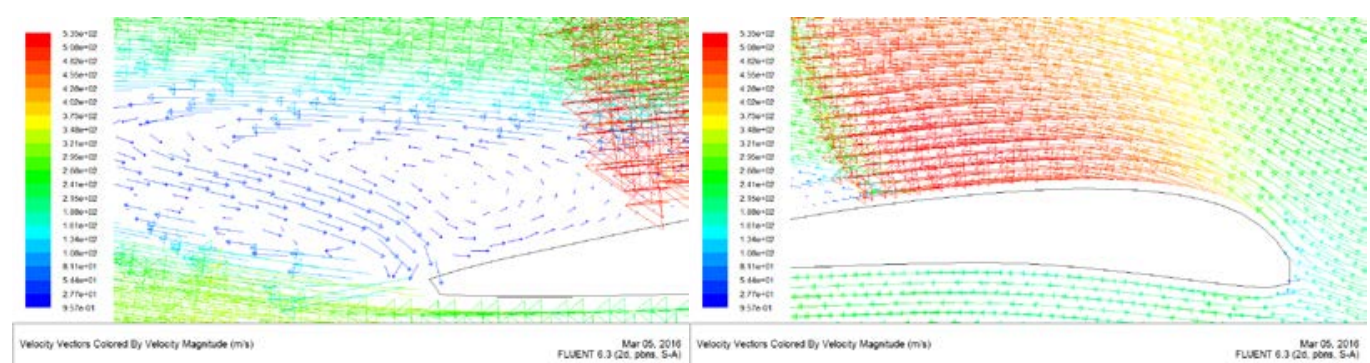

Fig.10 detail view (trailing vortex) Fig.11 detail view (shock wave at the top)

From an enlarged view, gas on the airfoil surface is disturbed by strong compression, which leads to a shock wave appear in an intermediate position. After going through the shock wave, pressure increases, and speed decreases. Rear airfoil has undergone significant boundary layer separation, and vortex is generated. Lift coefficient increasing trend slows down and even declines, while the drag coefficient increased significantly and aerodynamic characteristics are greatly worsen.

\section{Conclusion}

The study result shows that lift coefficient and drag coefficient are both affected by the Angle of attack, and almost approximately obey the quadratic curve. We can select the appropriate operating point according to the characteristics of the different area of the curve, so that the wind energy 
utilization can be maximized. By comparison with the relevant literature, aerodynamic characteristics obtained in this study is consistent with the experimental curve trend, indicating that the given aerodynamic characteristics of airfoil can be accurately simulated and predicted by using FLUENT numerical simulation and SA model which also can be viewed as important means of designing and testing wind turbine design in the future.

\section{References}

[1] Yongkang Chen. the research of airfoil aerodynamic characteristics of wind turbine blade in the horizontal axis[D].Harbin Institute of Technology, 2009

[2] Linjing Ma, Jiang Chen, Gang Du, Renjing Cao. the review of wind turbine blade numerical simulation[J].Acta Energiae Solaris Sinica,2010,02:203-209.

[3] Dan Liu, Junxiang Li, Zhongmin Xue, Chun Chen.the numerical simulation of wind turbine airfoil aerodynamic characteristics[J].Electrical Engineering, 2010,07:7-11+18. 